\title{
SOME PROPERTIES OF WEAK FORM OF $\gamma$-SEMI-OPEN SETS
}

\author{
SABIR HUSSAIN $^{1}$ AND MOHAMMAD AHMAD ALGHAMDI ${ }^{2}$
}

\begin{abstract}
In this paper, we introduce and explore fundamental properties of weak form of $\gamma$-semi-open sets namely maximal $\gamma$-semi-open sets in topological spaces such as decomposition theorem for maximal $\gamma$-semi-open set. Basic properties of intersection of maximal $\gamma$-semi-open sets are established, such as the $\gamma$-semi-closure law of $\gamma$-semiradical.
\end{abstract}

\section{Introduction}

Topology is an important and interesting area of mathematics, the study of which will not only introduce you to new concepts and theorems but also put into context old ones like continuous functions. It is so fundamental that its influence is evident in almost every other branch of mathematics. This makes the study of topology relevant to all who aspire to be mathematicians whether their first love is algebra, analysis, category theory, chaos, continuum mechanics, dynamics, geometry, industrial mathematics, mathematical biology, mathematical economics, mathematical finance, mathematical modelling, mathematical physics, mathematics of communication, number theory, numerical mathematics, operation research or statistics.

S. Kasahara [11] introduced and discussed an operation $\gamma$ of a topology $\tau$ into the power set $\mathrm{P}(\mathrm{X})$ of a space X. H. Ogata [13], introduced the concept of $\gamma$-open sets and investigated the related topological properties of the associated topology $\tau_{\gamma}$ and $\tau$ by using operation $\gamma$. So far several researchers worked on the findings of $\mathrm{H}$. Ogata and a lot of material is available in the literature.

S. Hussain and B. Ahmad [1-10] continued studying the properties of $\gamma$-operations on topological spaces and investigated many interesting results. N. Levine [12] introduced the notion of semi-open sets in topological spaces. Several topologist generalized many classical notions of topology using semi open sets introduced by Levine. Recently, S. Hussain, B. Ahmad and T. Noiri[10] introduced $\gamma$-semi-open sets in topological spaces as a generalization

Corresponding author: Sabir Hussain.

2010 Mathematics Subject Classification. 54A05, 54A10, 54D10, 54D99.

Key words and phrases. $\gamma$-semi-closed (open), $\gamma$-semi-closure(interior) , maximal $\gamma$-semi-open, pre- $\gamma$ semi-open sets, $\gamma$-semi-radical. 
of $\gamma$-open sets introduced by $H$. Ogata [13]. Moreover, they showed [10] that the concepts of $g \Lambda_{s}$-sets; $g \Lambda^{s}$-sets, semi- $T_{1}$ space and semi- $R_{0}$ space can be generalized by replacing the semi- open sets with $\gamma$-semi-open sets. Further B. Ahmad and S. Hussain [2] explored $\gamma$-semiinterior (closure) and $\gamma$-semi-continuous functions. They introduced and discussed minimal $\gamma$-semi-open sets in topological spaces [7] and obtained some properties of pre $\gamma$-semi-open sets using properties of minimal $\gamma$-semi-open sets. As an application of a theory of minimal $\gamma$-semi-open sets, they established a sufficient condition for a $\gamma$-semi-locally finite space to be a pre $\gamma$-semi- $T_{2}$ space.

Although the definition of the maximal $\gamma$-semi-open set is obtained by dualizing the definition of the minimal $\gamma$-semi-open set, the properties of them are quite different, especially the results in the last two sections. The purpose of this paper is to prove some fundamental properties of maximal $\gamma$-semi-open sets and establish a part of the foundation of the theory of maximal $\gamma$-semi-open sets in topological spaces.

First, we recall some definitions and results used in this paper. Hereafter, we shall write a space in place of a topological space.

\section{Preliminaries}

Throughout the present paper X denotes the topological space.

Definition([11]). An operation $\gamma: \tau \rightarrow P(X)$ is a function from $\tau$ to the power set of $\mathrm{X}$ such that $V \subseteq V^{\gamma}$, for each $V \in \tau$, where $V^{\gamma}$ denotes the value of $\gamma$ at $V$. The operations defined by $\gamma(\mathrm{G})=\mathrm{G}, \gamma(\mathrm{G})=\operatorname{cl}(\mathrm{G})$ and $\gamma(\mathrm{G})=\operatorname{intcl}(\mathrm{G})$ are examples of operation $\gamma$.

Definition.([11]). Let $A \subseteq X$. A point $\in A$ is said to be a $\gamma$-interior point of $A$, if there exists an open $n b d N$ of $x$ such that $N^{\gamma} \subseteq A$ and we denote the set of all such points by $i n t_{\gamma}(A)$. Thus

$$
\operatorname{int}_{\gamma}(A)=\left\{x \in A: x \in N \in \tau \text { and } N^{\gamma} \subseteq A\right\} \subseteq A \text {. }
$$

Note that $\mathrm{A}$ is $\gamma$-open [13] iff $\mathrm{A}=i n t_{\gamma}(\mathrm{A})$. A set $A$ is called $\gamma$ - closed [1] iff $X$ - $A$ is $\gamma$-open.

Definition([13]). A point $x \in X$ is called a $\gamma$-closure point of $A \subseteq X$, if $U^{\gamma} \cap A \neq \phi$, for each open $n b d U$ of $x$. The set of all $\gamma$-closure points of $A$ is called $\gamma$-closure of $A$ and is denoted by $c l_{\gamma}(A)$. A subset $A$ of $X$ is called $\gamma$-closed, if $c l_{\gamma}(A) \subseteq A$. Note that $c l_{\gamma}(A)$ is contained in every $\gamma$-closed superset of $A$.

Definition([10]). A subset $A$ of a space $(X, \tau)$ is said to be a $\gamma$-semi-open set, if there exists a $\gamma$-open set $\mathrm{O}$ such that $O \subseteq A \subseteq c l_{\gamma}(O)$. The set of all $\gamma^{*}$-semi-open sets is denoted by $S O_{\gamma(X)}$. $A$ is $\gamma$-semi-closed iff $X-A$ is $\gamma$-semi-open in $X$.

Definition.([5]). Let $A$ be a subset of a space $X$. The intersection of all $\gamma$-semi-closed sets containing $A$ is called $\gamma$-semi-closure of $A$ and is denoted by $\operatorname{scl}_{\gamma}(A)$.

Note that $A$ is $\gamma$-semi-closed if and only if $\operatorname{scl} l_{\gamma}(A)=A$. 
Definition([5]). Let $A$ be a subset of a space $X$. The union of $\gamma$-semi-open subsets of $A$ is called $\gamma$-semi-interior of $A$ and is denoted by $\operatorname{sint}_{\gamma}(A)$.

Definition.([5]) An operation $\gamma$ on $\tau$ is said be semi-regular, if for any semi-open $n b d s U, V$ of $x \in X$, there exists an semi-open nbd $W$ of $x$ such that $U^{\gamma} \cap V^{\gamma} \supseteq W^{\gamma}$.

Definition.([5]) An operation $\gamma$ on $\tau$ is said be semi-open, if for every $n b d U$ of $x \in X$, there exists a semi-open set $B$ such that $x \in B$ and $U^{\gamma} \supseteq B$.

Definition([6]) A space $X$ is said to be $\gamma$-semi- $T_{2}$, if for each distinct points $x, y \in X$, there exist semi-open sets $U, V$ such that $x \in U, y \in V$ and $U^{\gamma} \cap V^{\gamma}=\phi$.

\section{Maximal $\gamma$-semi-open sets}

Definition 3.1. A proper nonempty $\gamma$-semi-open subset $B$ of $X$ is said to be a maximal $\gamma$ semi-open set, if any $\gamma$-semi-open set which contains $B$ is $X$ or $B$.

Example 3.2. Let $X=\{a, b, c\}, \tau=\{\phi, X,\{a\},\{b\},\{a, b\},\{a, c\}\}$ be a topology on $X$. For $b \in X$, define an operation $\gamma: \tau \rightarrow P(X)$ by

$$
\gamma(A)=A^{\gamma}=\left\{\begin{array}{cc}
c l(A), & \text { if } b \in A \\
A, & \text { if } b \notin A
\end{array}\right.
$$

Calculation gives that $\gamma$-semi-open sets are $\{a\},\{a, b\},\{a, c\}, X, \phi$. Clearly $\{a, b\},\{a, c\}$ and $X$ are maximal $\gamma$-semi-open sets.

The following Lemma is straightforward from the Definition 3.1:

Lemma 3.3. (a) Let $U$ be a maximal $\gamma$-semi-open set and $W$ a $\gamma$-semi-open set. Then $U \cup W=$ $X$ or $W \subseteq U$.

(b) Let $U$ and $V$ be maximal $\gamma$-semi-open sets. Then $U \cup V=X$ or $U=V$.

The following is immediate:

Proposition 3.4. Let $U$ be a maximal $\gamma$-semi-open set. If $x \in U$, then $W \cup U=X$ or $W \subseteq U$, for any $\gamma$-semi-open nbd $W$ of $x$.

Theorem 3.5. Let $U_{\alpha}, U_{\beta}$ and $U_{\gamma}$ be maximal $\gamma$-semi-open sets such that $U_{\alpha} \neq U_{\beta}$. If $U_{\alpha} \cap U_{\beta} \subseteq$ $U_{\gamma}$, then $U_{\alpha}=U_{\gamma}$ or $U_{\beta}=U_{\gamma}$.

Proof. We see that

$$
\begin{aligned}
U_{\alpha} \cap U_{\gamma} & =U_{\alpha} \cap\left(U_{\gamma} \cap X\right)=U_{\alpha} \cap\left(U_{\gamma} \cap\left(U_{\alpha} \cup U_{\beta}\right)\right) \quad \text { (by Lemma 3.3(b)) } \\
& =U_{\alpha} \cap\left(\left(U_{\gamma} \cap U_{\alpha}\right) \cup\left(U_{\gamma} \cap U_{\beta}\right)\right)=\left(U_{\alpha} \cap U_{\gamma}\right) \cup\left(U_{\gamma} \cap U_{\alpha} \cap U_{\beta}\right) \\
& =\left(U_{\alpha} \cap U_{\gamma}\right) \cup\left(U_{\alpha} \cap U_{\beta}\right) \quad\left(\text { by } U_{\alpha} \cap U_{\beta} \subseteq U_{\gamma}\right)
\end{aligned}
$$




$$
=U_{\alpha} \cap\left(U_{\gamma} \cup U_{\beta}\right) .
$$

Hence we have $U_{\alpha} \cap U_{\gamma}=U_{\alpha} \cap\left(U_{\gamma} \cup U_{\beta}\right)$. If $U_{\gamma} \neq U_{\beta}$, then $U_{\gamma} \cup U_{\beta}=X$, and hence $U_{\alpha} \cap U_{\gamma}=U_{\alpha}$ implies $U_{\alpha} \subseteq U_{\gamma}$. Since $U_{\alpha}$ and $U_{\gamma}$ are maximal $\gamma$-semi-open sets, we have $U_{\alpha}=U_{\gamma}$. Hence the proof.

The following Theorem gives a relationship among maximal $\gamma$-semi-open sets:

Proposition 3.6. Let $U_{1}, U_{2}$ and $U_{3}$ be disjoint maximal $\gamma$-semi-open sets. Then, $U_{1} \cap U_{2} \nsubseteq$ $U_{1} \cap U_{3}$.

Proof. If $U_{1} \cap U_{2} \subseteq U_{1} \cap U_{3}$, then we see that $\left(U_{1} \cap U_{2}\right) \cup\left(U_{2} \cap U_{3}\right) \subseteq\left(U_{1} \cap U_{3}\right) \cup\left(U_{2} \cap U_{3}\right)$. Therefore $U_{2} \cap\left(U_{1} \cup U_{3}\right) \subseteq\left(U_{1} \cup U_{2}\right) \cap U_{3}$. Since $U_{1} \cup U_{3}=X=U_{1} \cup U_{2}$, we have $U_{2} \subseteq U_{3}$. It follows that $U_{2}=U_{3}$. This contradiction completes the proof.

Proposition 3.7. Let $U$ be a maximal $\gamma$-semi-open set and $x \in U$. Then $U=\cup\{W: W$ is $a$ $\gamma$-semi-open nbd of $x$ such that $W \cup U \neq X\}$, where $\gamma$ is a semi-regular operation.

Proof. This follows from Proposition 3.4 and the fact that $\mathrm{U}$ is a $\gamma$-semi-open nbh of $x$ [2]. Hence the proof.

Recall that the complement of any finite subset is a cofinite subset. The following Proposition shows the existence of maximal $\gamma$-semi-open sets for special cases.

Proposition 3.8. Let $V$ be a proper nonempty cofinite $\gamma$-semi-open subset. Then there exists, at least, one (cofinite) maximal $\gamma$-semi-open set $U$ such that $V \subseteq U$.

Proof. If $V$ is a maximal $\gamma$-semi-open set, we may set $U=V$. If $V$ is not a maximal $\gamma$-semiopen set, then there exists (cofinite) $\gamma$-semi-open set $V_{1}$ such that $V \subseteq V_{1} \neq X$. If $V_{1}$ is a maximal $\gamma$-semi-open set, we may set $U=V_{1}$. If $V_{1}$ is not a maximal $\gamma$-semi-open set, then there exists a (cofinite) $\gamma$-semi-open set $V_{2}$ such that $V \subseteq V_{1} \subseteq V_{2} \neq X$ Continuing this process, we have a sequence of $\gamma$-semi-open sets

$$
V \subseteq V_{1} \subseteq V_{2} \cdots \subseteq V_{k} \subseteq \cdots
$$

Since $V$ is a cofinite set, this process ends in a finite number of steps. Finally, we get a maximal $\gamma$-semi-open set $U=V_{n}$, for some positive integer $n$. This completes the proof.

\section{Applications of $\gamma$-semi-closure, $\gamma$-semi-interior in maximal $\gamma$-semi-open sets}

Now we study some relationship among $\gamma$-semi-closure, $\gamma$-semi-interior and a maximal $\gamma$-semi-open set. As an application, we prove Theorem 4.12 about pre $\gamma$-semi-open sets:

Theorem 4.1. Let $U$ be a maximal $\gamma$-semi-open set and $x \in(X-U)$. Then, $(X-U) \subseteq W$, for any $\gamma$-semi-open nbd $W$ of $x$. 
Proof. Since $x \in(X-U)$, we have $W \nsubseteq U$, for any $\gamma$-semi-open nbd W of $x$. Then $W \cup U=X$, by Lemma 3.3(a). Therefore $(X-U) \subseteq W$. This completes the proof.

Corollary 4.2. Let $U$ be a maximal $\gamma$-semi-open set. Then, either of the following holds:

(a) $W=X$, for each $x \in(X-U)$ and each $\gamma$-semi-open nbd $W$ of $x$.

(b) there exists a $\gamma$-semi-open set $W$ such that $(X-U) \subseteq W$ and $W \subseteq X$.

Proof. If (a) does not hold, then there exists an element $x \in(X-U)$ and a $\gamma$-semi-open nbd $W$ of $x$ such that $W \subseteq X$. By Theorem 4.1, we have $(X-U) \subseteq W$. Hence the proof.

Corollary 4.3. Let $U$ be a maximal $\gamma$-semi-open set. Then, either of the following holds:

(a) $(X-U) \subseteq W$, for each $x \in(X-U)$ and each $\gamma$-semi-open nbd $W$ of $x$.

(b) there exists a $\gamma$-semi-open set $W$ such that $(X-U)=W \neq X$.

Proof. Assume that (b) does not hold. Then by Theorem 4.1, we have $(X-U) \subseteq W$, for each $x \in(X-U)$ and each $\gamma$-semi-open nbd W of $\mathrm{x}$. Hence we have $(X-U) \subseteq W$. This completes the proof.

Corollary 4.4. Let $U$ be a maximal $\gamma$-semi-open set. Then $\operatorname{scl}_{\gamma}(U)=X \operatorname{or~scl}_{\gamma}(U)=U$.

Proof. Since U is a maximal $\gamma$-semi-open set. Then by Corollary 4.3, the following two cases hold:

(a) for each $x \in(X-U)$ and each $\gamma$-semi-open nbd $W$ of $x$, we have $(X-U) \subseteq W$. Since $(X-U) \neq W^{\gamma}$, we have $W \cap U \neq \phi$, for any $\gamma$-semi-open nbd and hence open $n b d W$ of $x$. Hence, $(X-U) \subseteq s c l_{\gamma}(U)$. Since $X=U \cup(X-U) \subseteq U \cup s c l_{\gamma}(U)=s c l_{\gamma}(U) \subseteq X$, we have $\operatorname{scl}_{\gamma}(U)=X$

(b) there exists a $\gamma$-semi-open set W such that $(X-U)=W \neq X$, since $(X-U)=W$ is a $\gamma$-semiopen set, $\mathrm{U}$ is a $\gamma$-semi-closed set. Therefore, $U=s c l_{\gamma}(U)$. This completes the proof.

The following Corollary follows from Corollary 4.4:

Corollary 4.5. Let $U$ be a maximal $\gamma$-semi-open set. Then $\operatorname{sint} t_{\gamma}(X-U)=(X-U)$ or $\operatorname{sint} t_{\gamma}(X-$ $U)=\phi$.

Theorem 4.6. Let $U$ be a maximal $\gamma$-semi-open set and $\phi \neq S \subseteq(X-U)$. Then $\operatorname{scl} l_{\gamma}(S)=(X-U)$.

Proof. Since $\phi \neq S \subseteq(X-U)$, then by Theorem 4.1, we have $W \cap S \neq \phi$, for any $x \in(X-U)$ and any $\gamma$-semi-open $n b d W$ of $x$. Then $(X-U) \subseteq s c l_{\gamma}(S)$. Since $X-U$ is a $\gamma$-semi-closed set and $S \subseteq(X-U)$, we see that $\operatorname{scl}_{\gamma}(S) \subseteq \operatorname{scl}_{\gamma}(X-U)=X-U$. Therefore, $X-U=s c l_{\gamma}(S)$. Hence the proof.

Corollary 4.7. Let $U$ be a maximal $\gamma$-semi-open set and $M \subseteq X$ with $U \subseteq M$. Then $\operatorname{scl}_{\gamma}(M)=X$. Where $\gamma$ is a semi-regular operation. 
Proof. Since $U \subseteq M \subseteq X$, there exists a $\phi \neq S \subseteq(X-U)$ such that $M=U \cup S$. By semi-regularity of operation $\gamma$ and Theorem 4.6, we have $s c l_{\gamma}(M)=s c l_{\gamma}(S \cup U)=\operatorname{scl}_{\gamma}(S) \cup s c l_{\gamma}(U) \supseteq(X-U) \cup$ $U=X$. Therefore $\operatorname{scl}_{\gamma}(M)=X$. This completes the proof.

The following Theorem follows from Corollary 4.7:

Theorem 4.8. Let $U$ be a maximal $\gamma$-semi-open set and $(X-U)$ has at least two elements. Then, for any element $a \in(X-U), \operatorname{scl}_{\gamma}(X-\{a\})=X$, where $\gamma$ is a semi-regularoperation.

Theorem 4.9. Let $U$ be a maximal $\gamma$-semi-open set and $U \subseteq N \subseteq X$. Then $\operatorname{sint}_{\gamma}(N)=U$.

Proof. If $N=U$, then $\operatorname{sint}_{\gamma}(N)=\operatorname{sint}_{\gamma}(U)=U$. Otherwise $N \neq U$, and hence $U \subseteq N$. It follows that $U \subseteq \operatorname{sint}_{\gamma}(N)$. Since $U$ is a maximal $\gamma$-semi-open set, we have also $\sin t_{\gamma}(N) \subseteq U$. Therefore $\operatorname{sint}_{\gamma}(N)=U$. Hence the proof.

The following Theorem follows from Theorems 4.6 and 4.9:

Theorem 4.10. Let $U$ be a maximal $\gamma$-semi-open set and $\phi \neq S \subseteq(X-U)$. Then, $X-s c l_{\gamma}(S)=$ int $_{\gamma}(X-S)=U$.

Definition 4.11 ([2]). A subset $M$ of $X$ is said to be pre- $\gamma$-semi-open-set, if $M \subseteq \operatorname{sint} t_{\gamma}\left(\operatorname{scl}_{\gamma}(M)\right)$.

The following result follows from Corollary 4.7.

Theorem 4.12. Let $U$ be a maximal $\gamma$-semi-open set and $U \subseteq M \subseteq X$. Then $M$ is a pre- $\gamma$-semiopen set, where $\gamma$ is a semi-regular operation.

Proof. If $M=U$, then $\mathrm{M}$ is a $\gamma$-semi-open set. Therefore $\mathrm{M}$ is a pre- $\gamma$-semi-open set [2]. Otherwise $U \subseteq M$, then by Corollary 4.7, $\operatorname{sint}_{\gamma}\left(\operatorname{scl}_{\gamma}(M)\right)=\operatorname{sint}_{\gamma}(X)=X \supseteq M$. Therefore $M$ is a pre- $\gamma$-semi-open set. Hence the proof.

The following Corollary directly follows from Theorem 4.12:

Corollary 4.13. Let $U$ be a maximal $\gamma$-semi-open set. Then $X-\{a\}$ is a pre- $\gamma$-semi-open set, for any $a \in(X-U)$, where $\gamma$ is a semi-regular operation.

\section{Basic properties of $\gamma$-semi-radical}

In this section, we prove fundamental properties of radical of maximal $\gamma$-semi-open sets. We establish a very useful decomposition theorem for a maximal $\gamma$-semi-open set in Theorem 5.7 .

Definition 5.1. Let $U=\left\{U_{\lambda}: \lambda \in I\right\}$ be a class of maximal $\gamma$-semi-open sets. Then $\cap U=$ $\bigcap_{\lambda \in I} U_{\lambda}$ is called the $\gamma$-semi-radical of $U$.

Example 5.2. Clearly is Example 3.2, the $\gamma$-semi-radical is the set $\{a\}$. 
The Symbol $I \backslash \Gamma$ means difference of index sets; namely, $I \backslash \Gamma=I-\Gamma$, and the cardinality of a set $I$ is denoted by $|I|$ in the following arguments:

Theorem 5.3. Suppose that $|I| \geq 2$. Let $U_{\lambda}$ be a maximal $\gamma$-semi-open set for any $\lambda \in I$ and $U_{\lambda} \neq U_{\mu}$, for any $\lambda, \mu \in I$ with $\lambda \neq \mu$. If $\mu \in I$, then the following hold:

(a) $X-\bigcap_{\lambda \in I-\{\mu\}} U_{\lambda} \subseteq U_{\mu}$.

(b) $\cap \bigcap_{\lambda \in I-\{\mu\}} U_{\lambda} \neq \phi$.

\section{Proof.}

(a) By Lemma 3.3(b), we have $\left(X-U_{\mu}\right) \subseteq U_{\lambda}$ for any $\lambda \in I$ with $\lambda \neq \mu$. Then $\left(X-U_{\mu}\right) \subseteq$ $\bigcap_{\lambda \in I-\{\mu\}} U_{\lambda}$. Therefore, we have $X-\bigcap_{\lambda \in I-\{\mu\}} U_{\lambda} \subseteq U_{\mu}$.

(b) If $\bigcap_{\lambda \in I-\{\mu\}} U_{\lambda}=\phi$. By (a), we have $X=U_{\mu}$. This is contradiction to our supposition that $U_{\lambda}$ is a maximal $\gamma$-semi-open set. Therefore, we have $\bigcap_{\lambda \in I-\{\mu\}} U_{\lambda} \neq \phi$. This completes the proof.

The following Corollary follows from Theorem 5.3(b):

Corollary 5.4. Let $U_{\lambda}$ be a maximal $\gamma$-semi-open set, for any $\lambda \in I$ and $U_{\lambda} \neq U_{\mu}$, for any $\lambda, \mu \in I$ with $\lambda \neq \mu$. If $|I| \geq 2$, then $U_{\lambda} \cap U_{\mu} \neq \phi$, for any $\lambda, \mu \in I$ with $\lambda \neq \mu$.

Theorem 5.5. Let $U_{\lambda}$ be a maximal $\gamma$-semi-open set for any $\lambda \in I$ and $U_{\lambda} \neq U_{\mu}$, for any $\lambda, \mu \in I$ with $\lambda \neq \mu$. Assume that $|I| \geq 2$. If $\mu \in$ I. Then, $\bigcap_{\lambda \in I-\{\mu\}} U_{\lambda} \nsubseteq U_{\mu} \nsubseteq \bigcap_{\lambda \in I-\{\mu\}} U_{\lambda}$.

Proof. If $\bigcap_{\lambda \in I-\{\mu\}} U_{\lambda} \subseteq U_{\mu}$. Then by Theorem 5.3(b), we have $X=\left(X-\bigcap_{\lambda \in I-\{\mu\}} U_{\lambda}\right) \cup\left(\bigcap_{\lambda \in I-\{\mu\}} U_{\lambda}\right) \subseteq U_{\lambda}$. This is contradiction to our assumption. If $U_{\mu} \subseteq$ $\bigcap_{\lambda \in I-\{\mu\}} U_{\lambda}$, then we have $U_{\mu} \subseteq U_{\lambda}$ and hence $U_{\mu}=U_{\lambda}$ for any element $\lambda \in(I-\{\mu\})$. This contradicts our assumption that $U_{\mu} \neq U_{\lambda}$ when $\lambda \neq \mu$. Hence the proof.

Corollary 5.6. Let $U_{\lambda}$ be a maximal $\gamma$-semi-open set, for any $\lambda \in I$ and $U_{\lambda} \neq U_{\mu}$, for any $\lambda, \mu \in I$ with $\lambda \neq \mu$. If $\phi \neq \delta \subseteq I$, then $\bigcap_{\lambda \in I-\{\delta\}} U_{\lambda} \nsubseteq \bigcap_{\iota \in \delta} U_{\iota} \nsubseteq \bigcap_{\lambda \in I-\{\delta\}} U_{\lambda}$.

Proof. Let $\iota \in \delta$. By Theorem 5.5,

$$
\bigcap_{\lambda \in I-\{\delta\}} U_{\lambda}=\bigcap_{\lambda \in(I-(\{\delta\} \cup\{l\}))} U_{\lambda} \nsubseteq U_{i}
$$

Therefore, we see $\bigcap_{\lambda \in I-\{\delta\}} U_{\lambda} \nsubseteq \bigcap_{\iota \in \delta} U_{\iota}$. On the other hand, since $\bigcap_{\iota \in \delta} U_{l}=\bigcap_{\iota \in((I-(I-\{\delta\})))} U_{\iota} \nsubseteq$ $\bigcap_{\lambda \in I-\{\delta\}} U_{\lambda}$, we have $\bigcap_{\iota \in \delta} U_{\iota} \nsubseteq \bigcap_{\lambda \in I-\{\delta\}} U_{\lambda}$. This completes the proof.

Theorem 5.7. Let $U_{\lambda}$ be a maximal $\gamma$-semi-open set for any $\lambda \in I$ and $U_{\lambda} \neq U_{\mu}$, for any $\lambda, \mu \in I$ with $\lambda \neq \mu$. If $\phi \neq \delta \subseteq I$, then $\bigcap_{\lambda \in I} U_{\lambda} \nsubseteq \bigcap_{\iota \in \delta} U_{l}$.

Proof. By Corollary 5.6, we have $\bigcap_{\lambda \in I} U_{\lambda}=\left(\bigcap_{\lambda \in I-\delta} U_{\lambda}\right) \cap\left(\cap_{\iota \in I} U_{l}\right) \nsubseteq \bigcap_{l \in I} U_{l}$. Hence the proof. 
Theorem 5.8. [Decomposition Theorem for Maximal $\gamma$-semi-Open Set.] Let $|I| \geq 2$. If $U_{\lambda}$ be a maximal $\gamma$-semi-open set for any $\lambda \in I$ and $U_{\lambda} \neq U_{\mu}$, for any $\lambda, \mu \in I$ with $\lambda \neq \mu$. Then for any $\mu \in I$,

$$
U_{\mu}=\left(\bigcap_{\lambda \in I} U_{\lambda}\right) \bigcup\left(X-\bigcap_{\lambda \in I-\{\mu\}} U_{\lambda}\right) .
$$

Proof. Let $\mu \in I$. By Theorem 5.3(a), we have

$$
\begin{aligned}
\left(\bigcap_{\lambda \in I} U_{\lambda}\right) \bigcup\left(X-\bigcap_{\lambda \in I-\{\mu\}} U_{\lambda}\right) & =\left(\left(\bigcap_{\lambda \in I-\{\mu\}} U_{\lambda}\right) \bigcap U_{\mu}\right) \bigcup\left(X-\bigcap_{\lambda \in I-\{\mu\}} U_{\lambda}\right) \\
& =\left(\left(\bigcap_{\lambda \in I-\{\mu\}} U_{\lambda}\right) \bigcup\left(X-\bigcap_{\lambda \in I-\{\mu\}} U_{\lambda}\right)\right) \bigcap\left(U_{\mu} \bigcup\left(\left(X-\bigcap_{\lambda \in I-\{\mu\}} U_{\lambda}\right)\right)\right) \\
& =U_{\mu} \bigcup\left(X-\bigcap_{\lambda \in I-\{\mu\}} U_{\lambda}\right)=U_{\mu} .
\end{aligned}
$$

Therefore, we have $U_{\mu}=\left(\bigcap_{\lambda \in I} U_{\lambda}\right) \cup\left(X-\bigcap_{\lambda \in I-\{\mu\}} U_{\lambda}\right)$. Hence the proof.

We use Theorem 5.8 to prove the following:

Theorem 5.9. Let $I$ be a finite set and $U_{\lambda}$ be a maximal $\gamma$-semi-open set, for any $\lambda \in I$ and $U_{\lambda} \neq U_{\mu}$, for any $\lambda, \mu \in I$ with $\lambda \neq \mu$. If $\bigcap_{\lambda \in I} U_{\lambda}$ is a $\gamma$-semi-closed set, then $U_{\lambda}$ is a $\gamma$-semiclosed set, for any $\lambda \in I$, where $\gamma$ is a semi-regular operation.

Proof. By Theorem 5.8, we have $U_{\mu}=\left(\bigcap_{\lambda \in I} U_{\lambda}\right) \cup\left(X-\bigcap_{\lambda \in I-\{\mu\}} U_{\lambda}\right)=\left(\bigcap_{\lambda \in I} U_{\lambda}\right) \cup\left(\bigcup_{\lambda \in I-\{\mu\}}(X-\right.$ $\left.U_{\lambda}\right)$. Since $I$ is a finite set and $\gamma$ is semi-regular so, $\bigcup_{\lambda \in I-\{\mu\}}\left(X-U_{\lambda}\right)$ is a $\gamma$-semi-closed set [13]. Hence $U_{\lambda}$ is a $\gamma$-semi-closed set by our assumption. this completes the proof.

The following Theorem gives a sufficient condition for the set of all maximal $\gamma$-semi-open sets:

Theorem 5.10. Assume that $|I| \geq 2$. Let $U_{\lambda}$ be a maximal $\gamma$-semi-open set, for any $\lambda \in I$ and $U_{\lambda} \neq U_{\mu}$, for any $\lambda, \mu \in I$ with $\lambda \neq \mu$. If $\bigcap_{\lambda \in I} U_{\lambda}=\phi$, then $\left\{U_{\lambda}: \lambda \in I\right\}$ is the set of all maximal $\gamma$-semi-open sets of $X$.

Proof. If there exists another maximal $\gamma$-semi-open set $U_{v}$ of $\mathrm{X}$, which is not equal to $U_{\lambda}$, for any $\lambda \in I$, then $\phi=\bigcap_{\lambda \in I} U_{\lambda}=\bigcap_{\lambda \in(I \cup\{v\})-\{v\}} U_{\lambda}$. By Theorem 5.3(b), we see that $\bigcap_{\lambda \in(I \cup\{v\})-\} v\}} U_{\lambda}$ $\neq \phi$. This contradicts our assumption. Hence the proof.

Example 5.11. Let $X$ be a $\gamma$-semi- $T_{2}$ space or a cofinite space or a cocountable space. If each point $\{x\}$ is $\gamma$-semi-closed, then $X-\{a\}$ is a maximal $\gamma$-semi-open set for any $a \in X$. Moreover, we see that $\{X-\{a\}: a \in X\}$ is the set of all maximal $\gamma$-semi-open sets of $X$ by Theorem 5.10, since $\bigcap_{a \in X}(X-\{a\})=\phi$.

\section{More about $\gamma$-semi-radical of maximal $\gamma$-semi-open sets}

In this section, we study the $\gamma$-semi-closure of $\gamma$-semi-radical of maximal $\gamma$-semi-open sets, we begin with a proposition. 
Proposition 6.1. Let $U_{\lambda}$ be a set, for any $\lambda \in I$. If $s c l_{\gamma}\left(\bigcap_{\lambda \in I} U_{\lambda}\right)=X$, then $\operatorname{scl}_{\gamma}\left(U_{\lambda}\right)=X$, for any $\lambda \in I$.

Proof. We see that $X=s c l_{\gamma}\left(\bigcap_{\lambda \in I} U_{\lambda}\right) \subseteq s c l_{\gamma}\left(U_{\lambda}\right)$. It follows that $s c l_{\gamma}\left(U_{\lambda}\right)=X$, for any $\lambda \in I$. Hence the proof.

Theorem 6.2. Let $I$ be a finite set and $U_{\lambda}$ be a maximal $\gamma$-semi-open set for any $\lambda \in I$. If $s c l_{\gamma}\left(\bigcap_{\lambda \in I} U_{\lambda}\right) \neq X$, then there exists $\lambda \in I$ such that $s c l_{\gamma}\left(U_{\lambda}\right)=U_{\lambda}$, where $\gamma$ is a semi-regular and an semi-open operation.

Proof. Suppose that $\operatorname{scl}_{\gamma}\left(U_{\lambda}\right)=X$ for any $\lambda \in I$. Let $\mu \in I$. Since $\gamma$ is semi-open, so $\bigcap_{\lambda \in I-\{\mu\}} U_{\lambda}$ is a $\gamma$-semi-open set. Also semi-regularity of operation $\gamma$ implies that

$$
\begin{aligned}
\operatorname{scl}_{\gamma}\left(\bigcap_{\lambda \in I} U_{\lambda}\right) & =\operatorname{scl} l_{\gamma}\left(\left(\bigcap_{\lambda \in I-\{\{\mu\}} U_{\lambda}\right) \bigcap U_{\mu}\right)=\operatorname{scl} \gamma\left(\left(\bigcap_{\lambda \in I-\{\{\mu\}} U_{\lambda}\right)\right) \bigcap \operatorname{scl}_{\gamma}\left(U_{\mu}\right) \\
& \supseteq\left(\bigcap_{\lambda \in I-\{\{\mu\}} U_{\lambda}\right) \bigcap \operatorname{scl} l_{\gamma}\left(U_{\mu}\right)=\left(\bigcap_{\lambda \in I-\{\{\mu\}} U_{\lambda}\right) \bigcap X=\left(\bigcap_{\lambda \in I-\{\{\mu\}} U_{\lambda}\right) .
\end{aligned}
$$

Therefore, $s c l_{\gamma}\left(\bigcap_{\lambda \in I-\{\{\mu\}} U_{\lambda}\right) \subseteq \operatorname{scl}_{\gamma}\left(\operatorname{scl}_{\gamma}\left(\bigcap_{\lambda \in I} U_{\lambda}\right)\right)=s c l_{\gamma}\left(\bigcap_{\lambda \in I} U_{\lambda}\right)$, since $\gamma$ is semi-open. On the other hand, we see that $\bigcap_{\lambda \in I} U_{\lambda} \subseteq \bigcap_{\lambda \in I-\{\mu\}} U_{\lambda}$. It follows that $s c l_{\gamma}\left(\bigcap_{\lambda \in I} U_{\lambda}\right)=s c l_{\gamma}\left(\bigcap_{\lambda \in I-\{\mu\}}\right.$ $\left.U_{\lambda}\right)$. Then by induction on the element of $I$, we see that $s c l_{\gamma}\left(\cap_{\lambda \in I} U_{\lambda}\right)=s c l_{\gamma}\left(U_{\lambda}\right)=X$, for any $\lambda \in I$. This contradicts our assumption that $s c l_{\gamma}\left(\cap_{\lambda \in I} U_{\lambda}\right) \neq X$. Therefore, we see that there exists $\lambda \in I$ such that $s c l_{\gamma}\left(U_{\lambda}\right)=U_{\lambda}$. This completes the proof.

The following example shows that the condition that $I$ is finite is necessary in Theorem 6.2.

Example 6.3. Let $X=\Re^{n}$, the $n$-dimensional Euclidean space. Let $U_{x}=X-\{x\}$, for any $x \in X$. Then $U_{x}$ is a maximal $\gamma$-semi-open set and hence we have

$$
\operatorname{scl} l_{\gamma}\left(\bigcap_{x \in X} U_{x}\right)=\operatorname{scl} l_{\gamma}(\phi)=\phi \neq X
$$

However, $\operatorname{scl}_{\gamma}\left(U_{x}\right)=X$ for any $x \in X$.

The $\gamma$-semi-radical of maximal $\gamma$-semi-open sets have the following outstanding property:

Theorem 6.4. [The $\gamma$-Semi-Closure Law of $\gamma$-Semi-Radical] Let I be finite and $U_{\lambda}$ is a maximal $\gamma$-semi-open set for each $\lambda \in I$. Let $\Gamma \subseteq I$ such that

$$
\operatorname{scl}_{\gamma}\left(U_{\lambda}\right)=\left\{\begin{array}{c}
U_{\lambda}, \quad \text { for any } \lambda \in \Gamma \\
X, \quad \text { for any } \lambda \in I-\Gamma
\end{array}\right.
$$

Then, $\operatorname{scl}_{\gamma}\left(\bigcap_{\lambda \in I} U_{\lambda}\right)=\bigcap_{\lambda \in I} U_{\lambda}(=X$, if $\Gamma=\phi)$, where $\gamma$ is a semi-regular and an semi-open operation. 
Proof. If $I=\phi$, then we have the result by Theorem 6.2. Otherwise $I \neq \phi$, and hence we see that

$$
\begin{aligned}
\operatorname{scl} l_{\gamma}\left(\bigcap_{\lambda \in I} U_{\lambda}\right) & =\operatorname{scl} l_{\gamma}\left(\left(\bigcap_{\lambda \in \Gamma} U_{\lambda}\right) \bigcap\left(\bigcap_{\lambda \in I-\Gamma} U_{\lambda}\right)\right)=\operatorname{scl} l_{\gamma}\left(\left(\bigcap_{\lambda \in \Gamma} U_{\lambda}\right)\right) \bigcap \operatorname{scl} l_{\gamma}\left(\left(\bigcap_{\lambda \in I-\Gamma} U_{\lambda}\right)\right) \\
& \supseteq\left(\bigcap_{\lambda \in \Gamma} U_{\lambda}\right) \bigcap \operatorname{scl} l_{\gamma}\left(\bigcap_{\lambda \in I-\Gamma} U_{\lambda}\right)=\left(\bigcap_{\lambda \in \Gamma} U_{\lambda}\right) \bigcap X=\bigcap_{\lambda \in \Gamma} U_{\lambda} .
\end{aligned}
$$

By Theorem 6.2 and the fact that $\left(\bigcap_{\lambda \in \Gamma} U_{\lambda}\right)$ is a $\gamma$-semi-open set. Since $\gamma$ is semi-open [2], it follows that

$$
\operatorname{scl} l_{\gamma}\left(\bigcap_{\lambda \in I} U_{\lambda}\right)=\operatorname{scl} l_{\gamma}\left(s c l_{\gamma}\left(\bigcap_{\lambda \in I} U_{\lambda}\right)\right) \supseteq \operatorname{scl} l_{\gamma}\left(\bigcap_{\lambda \in \Gamma} U_{\lambda}\right) .
$$

On the other hand, we see that $\bigcap_{\lambda \in I} U_{\lambda} \subseteq \bigcap_{\lambda \in \Gamma} U_{\lambda}$, and hence $\operatorname{scl} l_{\gamma}\left(\bigcap_{\lambda \in I} U_{\lambda}\right) \subseteq \operatorname{scl}_{\gamma}\left(\bigcap_{\lambda \in \Gamma} U_{\lambda}\right)$. It follows that $\operatorname{scl} l_{\gamma}\left(\bigcap_{\lambda \in I} U_{\lambda}\right)=\operatorname{scl}_{\gamma}\left(\bigcap_{\lambda \in \Gamma} U_{\lambda}\right)$. The $\gamma$-semi-radical $\bigcap_{\lambda \in \lambda} U_{\lambda}$ is a $\gamma$-semi-closed set since $U_{\lambda}$ is a $\gamma$-semi-closed set for any $\lambda \in \Gamma$ by our assumption. Therefore, we see that $s c l_{\gamma}\left(\bigcap_{\lambda \in I} U_{\lambda}\right)=\bigcap_{\lambda \in \Gamma} U_{\lambda}$. This completes the proof.

\section{References}

[1] B. Ahmad and S. Hussain, Properties of $\gamma$-operations on topological spaces, Aligarh Bull. Math., 22(1) (2003), 45-51.

[2] B. Ahmad and S. Hussain, $\gamma$-semi-open sets in topological spaces II, Sotheast Asian Bull. Math., 34(5) (2010), 997-1008.

[3] B. Ahmad and S. Hussain, $\gamma$-convergence in topological space, Southeast Asian Bull. Math., 29(2005), $832-842$.

[4] B. Ahmad and S. Hussain, $\gamma^{*}$-regular and $\gamma$-normal space, Math. Today., 22(1) (2006), 37-44.

[5] B. Ahmad and S. Hussain: On $\gamma$-s-closed subspaces, Far East Jr. Math. Sci., 31 (2) (2008), 279-291.

[6] B. Ahmad, S. Hussain and T. Noiri, On some mappings in topological space, Eur. J. Pure Appl. Math., 1(2008), 22-29.

[7] S. Hussain and B. Ahmad, On minimal $\gamma$-semi-open sets, (Submitted).

[8] S. Hussain and B. Ahmad, On $\gamma$-s-closed spaces, Sci. Magna Jr., 3(4) (2007), 89-93.

[9] S. Hussain and B. Ahmad, On $\gamma$-s-regular spaces and almost $\gamma$-s-continuous functions, Lobackevskii. J. Math., 30(4)(2009), 263-268. DOI: 10.1134/51995080209040039.

[10] S. Hussain, B. Ahmad and T. Noiri, $\gamma$-semi-open sets in topological spaces, Asian Eur. J. Math., 3(3)(2010), 427-433. DOI: 10.1142/S1793557110000337.

[11] S. Kasahara, Operation-compact spaces, Math. Japon., 24(1979), 97-105.

[12] N. Levine, Semi-open sets and semi continuity in topological spaces, Amer. Math. Monthly, 70 (1963), 3641.

[13] H. Ogata: Operations on topological spaces and associated topology, Math. Japon., 36(1) (1991), $175-184$.

${ }^{1}$ Department of Mathematics, Yanbu University, P. O. Box 31387, Yanbu Alsinaiyah, Saudi Arabia.

Permanent Address: Department of Mathematics, Islamia University Bahawalpur, Pakistan.

E-mail: sabiriub@yahoo.com

${ }^{2}$ Department of Mathematics, King Abdul Aziz University, P. O. Box 80203, Jeddah 21589, saudi Arabia. E-mail: proff-malghamdi@hotmail.com 